\title{
Discrete flow pooling problems in coal supply chains
}

\author{
N. Boland ${ }^{\mathrm{a}}$, T. Kalinowski ${ }^{\mathrm{b}}$ and F. Rigterink ${ }^{\mathrm{b}}$ \\ ${ }^{\mathrm{a}}$ H. Milton Stewart School of Industrial and Systems Engineering, Georgia Institute of Technology \\ ${ }^{\mathrm{b}}$ School of Mathematical and Physical Sciences, The University of Newcastle, Australia \\ Email: fabian.rigterink@uon.edu.au
}

\begin{abstract}
The pooling problem is a nonconvex nonlinear programming problem (NLP) with applications in the refining and petrochemical industries, but also the coal mining industry. The problem can be stated as follows: given a set of raw material suppliers (inputs) and qualities of the supplies, find a cost-minimising way of blending these raw materials in intermediate pools and outputs so as to satisfy requirements on the output qualities. The blending in two stages (in pools and outputs) introduces bilinear constraints. The pooling problem can alternatively be described as a minimum cost network flow problem with additional bilinear constraints to capture the blending of raw materials.

In this paper we study a variation of the pooling problem that arises naturally in the coal mining industry and is sometimes referred to as grade targeting. Coal is made-to-order according to customers' desired product qualities. Deviations from these target qualities result in contractually agreed bonuses and penalties. In the pooling problem variation we study, costs are associated with these bonuses and penalties instead of network flows. While in the original pooling problem we have hard bounds on the qualities and unmet demand is penalised in the objective function, in our coal mining variation we have hard demand constraints and deviations from target qualities are penalised. This makes finding a feasible solution easy, while in the pooling problem finding a nontrivial feasible solution that satisfies the quality requirements is already hard. An implication of this is that we are able to solve larger problem instances than those typically studied in the pooling problem literature.

To model the coal blending process accurately, we define a time-expanded network where the intermediate pools represent coal stockpiles over time. Since coal is transported in large quantities, we study the trade-off between continuous and discretized flows in coal blending, i.e., solving a continuous flow problem where arbitrarily small flows are allowed versus solving a discretized flow problem where flows must be in multiples of some basic unit, e.g. trainloads. We also study two exact mixed-integer linear programming (MILP) linearizations of these mixed-integer nonlinear programs (MINLPs), which can be derived from unary and binary expansions of the flow integrality constraint. Such discretizations are typically studied as approximations to an originally continuous problem, however, in our application, a discretized formulation describes the original problem more accurately than a continuous formulation.
\end{abstract}

The paper is organized as follows. In Section 1.1, we introduce the pooling problem and present a variant of the well-known PQ-formulation. In Section 1.2, we extend the pooling problem to model a simplified coal supply chain. After a short literature review on coal supply chains, we present four different problems: the continuous flow problem (a MINLP), in which arbitrarily small flows are allowed, and three discretized flow problems (a MINLP and two MILPs), in which flows must be in multiples of trainloads. The discretization can be achieved by adding integrality constraints for the flow variables. We then show how to overcome the nonlinearity which is inherent in the pooling problem with the use of unary and binary expansions of the integer flow variables, which yields exact MILP reformulations of the discretized MINLP. We conclude the paper with Section 2 where we provide computational results for the four different problems which we solve for a real-life industry setting.

Keywords: Coal blending, pooling problem, mixed-integer nonlinear programming, mixed-integer linear programming 
Table 1. Notation

(a) Pooling problem

Sets

\begin{tabular}{rl}
$V$ & Set of vertices \\
$I$ & Set of inputs \\
$L$ & Set of pools \\
$J$ & Set of outputs \\
$A$ & Set of arcs \\
$\delta^{-}(v)$ & Set of incoming arcs of $v \in L \cup J$ \\
$\delta^{+}(v)$ & Set of outgoing arcs of $v \in I \cup L$ \\
$K$ & Set of qualities \\
& \\
Parameters & \\
\hline$\Lambda$ & Adjacency matrix of $G=(V, A)$ \\
$c_{a}$ & Per unit cost of flow on arc $a \in A$ \\
$\lambda_{v k}$ & Quality value of input $v \in I$ for quality $k \in K$ \\
$L_{v}^{V}, U_{v}^{V}$ & Lower and upper bound on total flow through \\
$L_{a}^{A}, U_{a}^{A}$ & $v \in V$ \\
$L_{v k}^{K}, U_{v k}^{K}$ & Lower and upper bound on $y_{a}, a \in A$ \\
& $v \in J$ for quality $k \in K$ \\
Variables & \\
\hline$y_{a}$ & Flow on arc $a \in A$ \\
$q_{i v}$ & Fraction of total flow through $v \in I \cup L$ that \\
& comes from input $i \in I$ \\
$q_{i a}$ & Fraction of $y_{a}, a \in A$, that comes from input \\
& $i \in I: q_{i a}=q_{i v}, a=(v, w) \in A, i \in I$ \\
$x_{i a}$ & Flow in $y_{a}, a \in A$, that comes from input $i \in I$ \\
& \\
\hline & \\
\hline &
\end{tabular}

(b) Coal supply chain extension

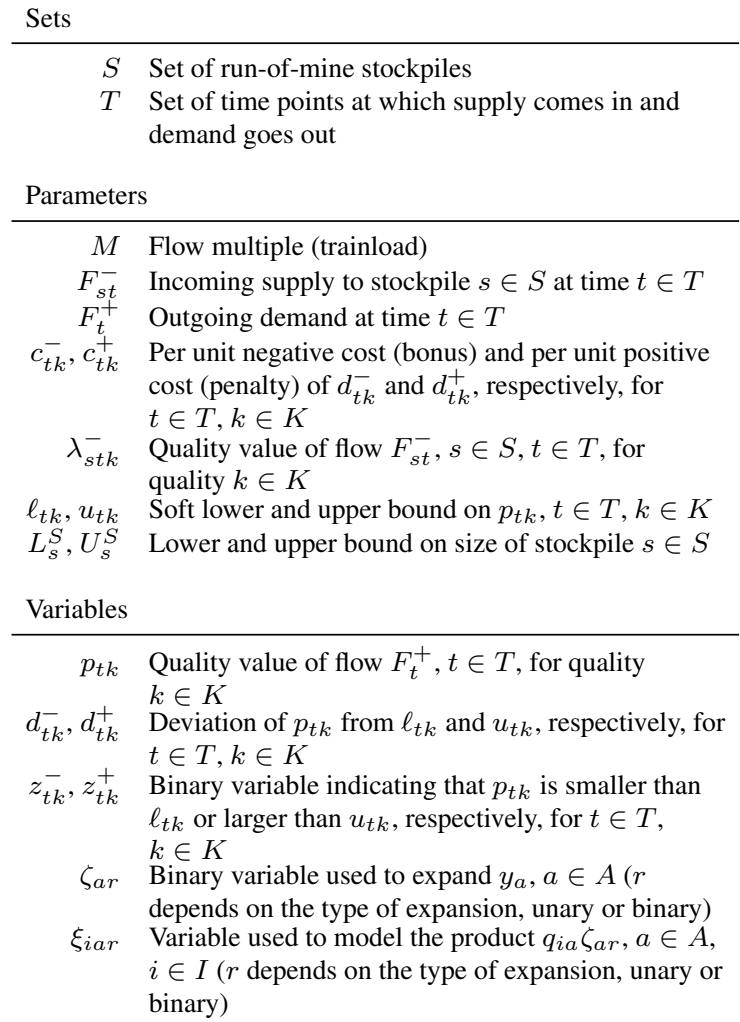

\section{Problem formulation}

\subsection{Pooling problem}

We consider a directed graph $G=(V, A)$ where $V$ is the set of vertices and $A$ is the set of arcs. $V$ is partitioned into three nonempty subsets $I, L, J \subset V: I$ is the set of inputs, $L$ is the set of pools and $J$ is the set of outputs. Flows are blended in pools and outputs. We assume that $A \subseteq(I \times L) \cup(L \times L) \cup(L \times J) \cup(I \times J)$, i.e., there are no arcs between two inputs $(A \cap(I \times I)=\emptyset)$ or two outputs $(A \cap(J \times J)=\emptyset)$ and no backward arcs from pools to inputs $(A \cap(L \times I)=\emptyset)$ or outputs to pools $(A \cap(J \times L)=\emptyset)$ or outputs to inputs $(A \cap(J \times I)=\emptyset)$. Throughout this paper, we write $A=A_{I L} \cup A_{L L} \cup A_{L J} \cup A_{I J}$ where $A_{I L}=A \cap(I \times L), A_{L L}=A \cap(L \times L)$, $A_{L J}=A \cap(L \times J)$, and $A_{I J}=A \cap(I \times J)$. We consider a set of qualities $K$ whose quality values are tracked across the network. We assume linear blending, i.e., the quality value of pools and outputs $v \in L \cup J$ for quality $k \in K$ is a linear combination of the incoming quality values weighted by the corresponding incoming flows as fractions of the total incoming flow. Instances with $A_{L L}=\emptyset$ are referred to as standard pooling problems (SPPs), and instances with $A_{L L} \neq \emptyset$ are referred to as generalized pooling problems (GPPs). Both SPPs and GPPs can be modelled as bilinear programs, which are special cases of quadratically constrained quadratic programs, which in turn are special cases of nonlinear programs. Instances with $L=\emptyset$ are referred to as blending problems, which can be modelled as linear programs.

For every pool and output $v \in L \cup J$, we denote the set of incoming $\operatorname{arcs}$ of $v$ by $\delta^{-}(v)$, and for every input and pool $v \in I \cup L$, we denote the set of outgoing arcs of $v$ by $\delta^{+}(v)$. Let $y_{a}$ be the flow on arc $a \in A$ and let $c_{a}$ be the corresponding per unit cost. The total flow through vertex $v \in V$ (resp. the flow on $\operatorname{arc} a \in A$ ) is bounded below by $L_{v}^{V}$ (resp. $L_{a}^{A}$ ) and above by $U_{v}^{V}$ (resp. $U_{a}^{A}$ ). For every input $i \in I$ and quality $k \in K$, the quality value of the incoming raw material is given by $\lambda_{i k}$. Similarly, for every output $v \in J$ and quality $k \in K$, the lower and upper bounds on the quality value of the outgoing blend are given by $L_{v k}^{K}$ and $U_{v k}^{K}$, respectively. Table 1 (a) summarises the notation for the pooling problem.

Significant differences in solution quality (when solved locally) and solve time (when solved locally or globally) can be seen when reformulating the pooling problem. Such reformulations typically use different (ag- 
gregating or disaggregating) variables and/or additional valid (but redundant) constraints. Recently, Alfaki and Haugland (2013) proposed a multi-commodity flow formulation for the pooling problem based on input commodities. Boland et al. (2015a) generalised these ideas and proposed new multi-commodity flow formulations based on output, input and output and (input, output)-commodities. Their computational results suggest that input and output commodities perform best, and since input commodities are more intuitive than output commodities, we now present the multi-commodity flow formulation based on input commodities, commonly referred to as the PQ-formulation.

The PQ-formulation uses fraction variables $q$, flow variables $y$ and disaggregated flow variables $x$, which are products of $q$ and $y$. Let $q_{i v}$ denote the fraction of total flow through $v \in I \cup L$ that comes from input $i \in I$. In particular, for $v \in I$ we have $q_{v v}=1$ and $q_{i v}=0$ for $i \in I \backslash\{v\}$. Since we assume linear blending, the fraction of $y_{a}, a=(v, w) \in A$, that comes from input $i \in I$ is equal to $q_{i v}$. For convenience, we introduce $q_{i a}=q_{i v}$ for all $a=(v, w) \in A$ and $i \in I$. Introducing both arc- and node-based fraction variables allows us to formulate both arc- and node-based constraints. Note, however, that the $q_{i a}$ do not appear in an implementation of the model, but are replaced by the corresponding $q_{i v}$. Lastly, let $x_{i a}$ denote the flow in $y_{a}$, $a \in A$, that comes from input $i \in I$. The PQ-formulation can be stated as follows:

$$
\begin{aligned}
& \text { [PQ] } \min _{q, x, y} \sum_{a \in A} c_{a} y_{a} \\
& \text { s.t. } \quad \sum_{a \in \delta^{-}(v)} y_{a}=\sum_{a \in \delta^{+}(v)} y_{a} \text {, } \\
& L_{v}^{V} \leq \sum_{a \in \delta^{+}(v)} y_{a} \leq U_{v}^{V}, \quad v \in I \cup L, \\
& L_{v}^{V} \leq \sum_{a \in \delta^{-}(v)} y_{a} \leq U_{v}^{V}, \quad v \in J, \\
& L_{a}^{A} \leq y_{a} \leq U_{a}^{A}, \\
& q_{i v} \geq 0, \\
& \sum_{i \in I} q_{i v}=1 \\
& \sum_{a \in \delta^{-}(v)}^{i \in I} x_{i a}=\sum_{a \in \delta^{+}(v)} x_{i a} \\
& L_{v k}^{K} \sum_{a \in \delta^{-}(v)}^{a \in \delta^{-}(v)} y_{a} \leq \sum_{i \in I}^{a \in \delta^{+}(v)} \sum_{a \in \delta^{-}(v)} \lambda_{i k} x_{i a} \leq U_{v k}^{K} \sum_{a \in \delta^{-}(v)} y_{a}, \quad v \in J, \quad k \in K, \\
& y_{a}=\sum_{i \in I} x_{i a} \\
& a \in A \\
& a \in A \text {, } \\
& v \in I \cup L, \quad i \in I \text {, } \\
& v \in I \cup L \text {, } \\
& v \in L, \quad i \in I, \\
& v \in L, \quad i \in I, \\
& a \in A, \quad i \in I .
\end{aligned}
$$

(1) is a flow conservation constraint which ensures that at every pool, the total incoming flow equals the total outgoing flow. (2) and (3) are vertex capacity constraints and (4) is an arc capacity constraint. Constraints (5) and (6) ensure that all fraction variables are between zero and one and that for every input and pool, the fraction variables sum to one. (7) can be interpreted as a disaggregated flow conservation constraint: while (1) ensures that at every pool, the total incoming flow must equal the total outgoing flow (regardless of the origin of the flows), (7) ensures that at every pool, the total incoming flow originating from a particular input must equal the total outgoing flow originating from the same input. It can be shown that (7) implies (1), i.e., that (1) is redundant. (8) is the output blending constraint. (9) and (10) are valid (but redundant) constraints. Adding such redundant constraints significantly improves the computational performance of a formulation. (11) outsources the $q y$ terms - which would otherwise appear in (7)-(10) - into a single constraint. The nonconvex nonlinearities of the pooling problem are only present in constraint (11). If we discretize e.g. the flow variable $y$ and expand it, then we can overcome the nonconvex nonlinearities by breaking the continuous bilinear constraint into a finite number of linear constraints, one of which is active through an SOS1 constraint. Note that by substituting (11) into (7)-(10), the PQ-formulation can also be stated without the $x$ variables.

\subsection{Coal supply chain extension}

Coal supply chains have been studied extensively in recent years. Liu and Kozan (2011) model a coal train scheduling problem as a Blocking Parallel-Machine Job-Shop Scheduling (BPMJSS) problem. Thomas et al. 


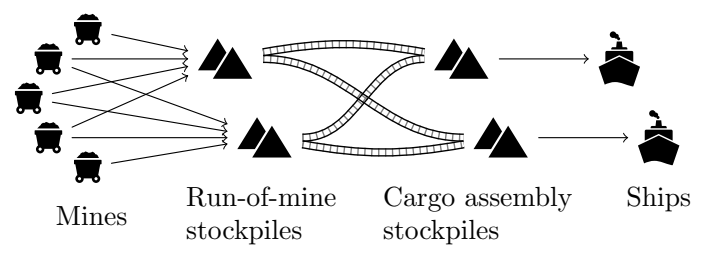

Figure 1. Simplified coal supply chain

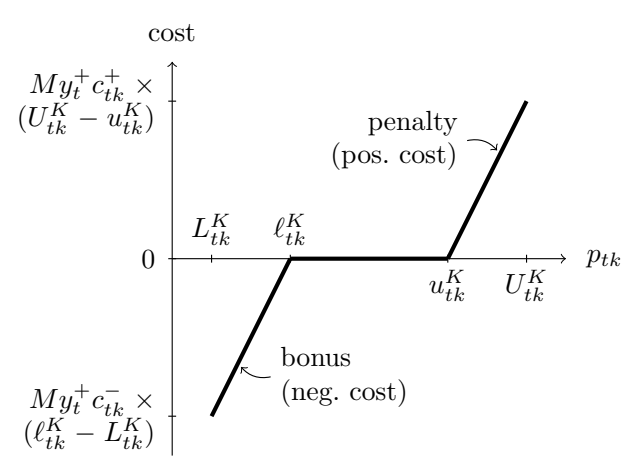

Figure 2. Bonus/penalty function

(2013) address an integrated planning and scheduling problem motivated by the coal supply chains in Australia. Both problems are NP-hard (Thomas et al. (2013)). Kozan and Liu (2012) propose a demand-responsive decision support system integrating the operations of coal shipment, stockpiles and railing. While these papers address short- to medium-term planning horizons, Singh et al. (2012) develop a large scale strategic capacity planning model for a coal supply chain. The aforementioned all propose MILPs that do not consider blending. Singh et al. (2014) develop an optimization tool for Rio Tinto's iron ore operations that does consider blending, addressing the nonlinearities using successive linear programming, a local optimization technique.

We consider the simplified coal supply chain shown in Figure 1. Coal is mined and loaded onto run-of-minestockpiles. It is then transported by rail to a cargo assembly terminal where it is offloaded onto stockpiles built for specific orders. The order-specific coal blends are then reclaimed and loaded onto ships. Contrary to such a pull system, there is a trend towards push systems and dedicated stockpile terminals (see Boland et al. (2015c)). However, in this paper, we only consider the former. We assume linear stockpile blending, which is an approximation of the actual stockpile blending behaviour. Formulations that model the blending behaviour more accurately (by predicting the geometry of a stockpile and taking into account the variability of the incoming quality values) can be found in Robinson (2004).

Let $S$ denote the set of run-of-mine stockpiles whose sizes are bounded below by $L_{s}^{S}$ and above by $U_{s}^{S}$ for stockpile $s \in S$, and let $T$ denote the set of time points at which orders/ships arrive. The preceding time point of $t \in T$ is $\tau(t):=\max \left\{t^{\prime} \in T \cup\{-\infty\}: t^{\prime}<t\right\}$. For every ship arriving at $t \in T$ (i.e., outgoing demand), we aggregate the coal that is mined and loaded onto run-of-mine stockpiles (i.e., incoming supply) during the time interval $(\tau(t), t]$. That is, for every $t \in T$, we have incoming supply to stockpile $s \in S$, denoted by $F_{s t}^{-}$, and outgoing demand, denoted by $F_{t}^{+}$. Between the run-of-mine stockpiles and the cargo assembly terminal, coal is transported in multiples of trainloads of size $M$ (e.g. 8 kilotonnes (kt)). We assume that incoming supply $F_{s t}^{-}$and outgoing demand $F_{t}^{+}$are given in multiples of $M$. The same applies to arc and vertex capacities. Every order $t \in T$ has soft and hard lower and upper bounds $L_{t k}^{K} \leq \ell_{t k} \leq u_{t k} \leq U_{t k}^{K}$ on the coal blend quality values, which are denoted by $p_{t k}$. We assume that smaller quality values are preferred (consider e.g. an unwanted coal quality such as sulfur content). If $p_{t k} \in\left[L_{t k}^{K}, \ell_{t k}\right]$, then the mining company is paid a per unit bonus (a negative cost) of $c_{t k}^{-}<0$ for the deviation $d_{t k}^{-}=\ell_{t k}-p_{t k} \geq 0$, i.e., we add $M F_{t}^{+} c_{t k}^{-} d_{t k}^{-}$ to the objective function. If $p_{t k} \in\left[u_{t k}, U_{t k}^{K}\right]$, then the mining company pays a per unit penalty (a positive cost) of $c_{t k}^{+}>0$ for the deviation $d_{t k}^{+}=p_{t k}-u_{t k} \geq 0$, i.e., we add $M F_{t}^{+} c_{t k}^{+} d_{t k}^{+}$to the objective function. If $p_{t k} \in\left(\ell_{t k}, u_{t k}\right)$, then neither a bonus nor a penalty is paid. Quality values $p_{t k} \in\left(-\infty, L_{t k}^{K}\right) \cup\left(U_{t k}^{K}, \infty\right)$ are infeasible. Table 1 (b) summarises the notation for the coal supply chain extension. The bonus/penalty function is shown in Figure 2.

As described in Boland et al. (2015b), we construct a time-expanded pooling problem network where inputs and pools are (run-of-mine stockpile, time point)-pairs $(s, t)$. The intermediate pools represent run-of-mine stockpiles over time. An output represents an order-specific cargo assembly stockpile. We model incoming supply and outgoing demand by setting the arc and vertex capacities appropriately. Since we have flow conservation constraint (11), we add a supersink output to account for the stockpile surplus.

As an example, consider the following problem, shown in part in Figure 3 . Throughout this example, a trainload is $M=8 \mathrm{kt}$ and there is only one quality, ash, measured in dry basis percent (db). Since there is only one quality, we omit the index $k$ in all parameters and variables. We have two stockpiles (i.e., $S=\{1,2\}$ ) with lower and upper bounds of 16 and $56 \mathrm{kt}$ for stockpile \#1 (i.e., $L_{1}^{S}=2$ and $U_{1}^{S}=7$ in multiples of 


\begin{tabular}{lll}
\hline Trainload & 8,000 & \\
Stockpile & Minimum & Maximum \\
$\# 1$ & 16,000 & 56,000 \\
$\# 2$ & 16,000 & 64,000 \\
\hline $2015-11-29$ & & \\
Supply & 40,000 & to \#1 \\
Supply & 48,000 & to \#2 \\
Demand & 32,000 & to ship \#1 \\
\hline 2015-12-04 & & \\
Supply & 16,000 & to \#1 \\
Supply & 24,000 & to \#2 \\
Demand & 32,000 & to ship \#2 \\
\hline
\end{tabular}

(a) Given data

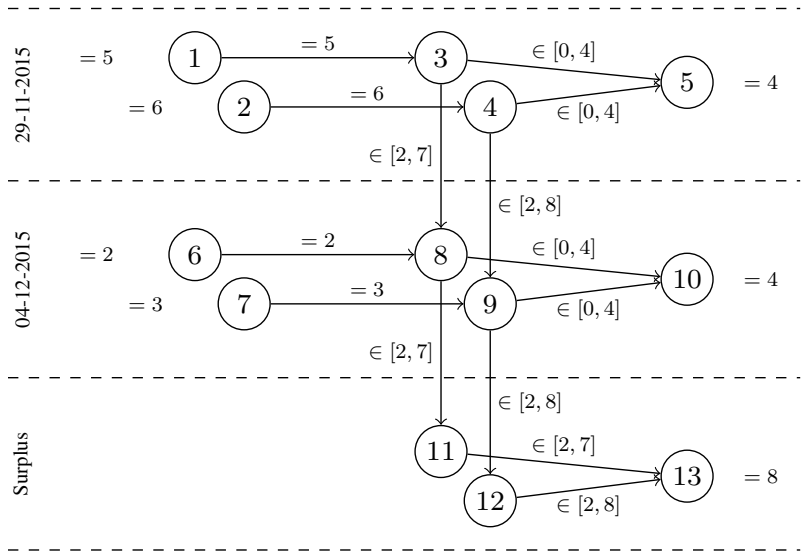

(b) Corresponding pooling problem

Figure 3. Example of given data (a) and the corresponding pooling problem (b).

$M$ ), and 16 and $64 \mathrm{kt}$ for stockpile \#2 (i.e., $L_{2}^{S}=2$ and $U_{2}^{S}=8$ in multiples of $M$ ). There are two time points at which supply comes in and demand goes out. For the first time point, we have supply of 40 and $48 \mathrm{kt}$, and demand of $32 \mathrm{kt}$ (i.e., $F_{11}^{-}=5, F_{21}^{-}=6$ and $F_{1}^{+}=4$ ). The quality values of the incoming supplies are $\lambda_{11}^{-}=10 \mathrm{db}$ and $\lambda_{21}^{-}=11 \mathrm{db}$, and the bounds on the quality value of the outgoing demand are $\left[L_{1}^{K}, \ell_{1}^{K}, u_{1}^{K}, U_{1}^{K}\right]=[7,8.5,9.5,11] \mathrm{db}$. The costs of deviation are $c_{1}^{-}=-5 \$ /(\mathrm{db} \cdot$ tonne $)$ and $c_{1}^{+}=10$ $\$ /(\mathrm{db} \cdot$ tonne $)$. For the second time point, we have $F_{12}^{-}=2, F_{22}^{-}=3, F_{2}^{+}=4, \lambda_{12}^{-}=8 \mathrm{db}, \lambda_{22}^{-}=7 \mathrm{db}$, $\left[L_{2}^{K}, \ell_{2}^{K}, u_{2}^{K}, U_{2}^{K}\right]=[7,8.5,9.5,11] \mathrm{db}, c_{2}^{-}=-5 \$ /(\mathrm{db} \cdot$ tonne $)$ and $c_{2}^{+}=10 \$ /(\mathrm{db} \cdot$ tonne $)$. Through the enumeration of all flow combinations, one can easily check that the solutions $y_{1}^{*}=((3,1),(0,4))$ and $y_{2}^{*}=((3,1),(1,3))$ are optimal (i.e., satisfying the first demand with flows of $3 M$ from stockpile \#1 and $1 M$ from \#2, and satisfying the second demand with flows of $0 M$ resp. $1 M$ from stockpile \#1 and $4 M$ resp. $3 M$ from \#2). The solutions have quality values of the outgoing demand of $p_{1}=10.25 \mathrm{db}$ and $p_{2}=9 . \overline{36} \mathrm{db}$ resp. $p_{2}=9 . \overline{45} \mathrm{db}$, with an objective of $M F_{1}^{+} c_{1}^{+} d_{1}^{+}=8 \mathrm{kt} \cdot 4 \cdot 10 \$ /(\mathrm{db} \cdot$ tonne $) \cdot(10.25-9.5) \mathrm{db}=240,000 \$$. These solutions are a recipe to the coal supply chain coordinator on how to optimally blend coal in order to minimize costs.

Continuous flow problem: In the continuous problem, arbitrarily small flows are allowed, i.e., we assume $y$ to be continuous. Let $z_{t k}^{-}$and $z_{t k}^{+}$be binary variables used in constraints (12)-(16) to model the bonus/penalty function shown in Figure 2. The problem can be stated as follows:

$$
\begin{aligned}
& \text { [C] } \min _{d, p, q, x, y, z} \sum_{t \in T} \sum_{k \in K} M F_{t}^{+}\left(c_{t k}^{-} d_{t k}^{-}+c_{t k}^{+} d_{t k}^{+}\right) \\
& \text {s.t. } \quad \text { (1)-11], }, \\
& z_{t k}^{-}, z_{t k}^{+} \in\{0,1\}, \\
& z_{t k}^{-}+z_{t k}^{+} \leq 1, \\
& p_{t k}=\frac{1}{F_{t}^{+}} \sum_{i \in I} \sum_{a \in \delta^{-}\left(v_{t}^{J}\right)} \lambda_{i k} x_{i a}, \\
&\left(\ell_{t k}-p_{t k}\right)^{+} \leq d_{t k}^{-} \leq \min \left\{z_{t k}^{-}\left(\ell_{t k}-L_{t k}^{K}\right),\left(\ell_{t k}-p_{t k}\right)+\left(1-z_{t k}^{-}\right)\left(U_{t k}^{K}-\ell_{t k}\right)\right\}, \\
&\left(p_{t k}-u_{t k}\right)^{+} \leq d_{t k}^{+} \leq \min \left\{z_{t k}^{+}\left(U_{t k}^{K}-u_{t k}\right),\left(p_{t k}-u_{t k}\right)+\left(1-z_{t k}^{+}\right)\left(u_{t k}-L_{t k}^{K}\right)\right\}, \\
& t \in T, k \in K .
\end{aligned}
$$

Discretized flow problems: In the discretized problem, flows must be in multiples of trainloads. This can be modelled by adding an integrality constraint for $y$ :

$$
\text { [D] } \min _{d, p, q, x, y, z} \sum_{t \in T} \sum_{k \in K} M F_{t}^{+}\left(c_{t k}^{-} d_{t k}^{-}+c_{t k}^{+} d_{t k}^{+}\right)
$$

s.t. (1)-16,

$$
y_{a} \in \mathbb{Z}, \quad a \in A \text {. }
$$

Modelling the unary and binary expansions of the integrality constraint (17), we can derive two exact linearizations of the nonlinear constraint (11), as described in Gupte et al. (2013). Let $\zeta_{a r}$ be the binary variables used 
in the expansion of $y_{a}$. We have $r \in\left\{0, \ldots, \bar{y}_{a}-\underline{y}_{a}\right\}$ for the unary and $r \in\left\{0, \ldots,\left\lfloor\log _{2}\left(\bar{y}_{a}-\underline{y}_{a}\right)\right\rfloor\right\}$ for the binary expansion of $y_{a}$. Finally, let $\xi_{i a r}$ be the continuous variable used to model the product $q_{i a} \zeta_{a r}$ for each $r$. The unary and binary expansions of [D], denoted by [D-U] and [D-B] respectively, both optimize over the variables $d, p, q, x, y, z, \xi, \zeta$, with the same objective function as in [D], and using constraints (1)-(10) and (12)-16. The remaining constraints for each formulation are as follows.

$$
\begin{aligned}
x_{i a} & =\sum_{r \in M_{a}}\left(\underline{y}_{a}+r\right) \xi_{i a r}, \quad q_{i a}=\sum_{r \in M_{a}} \xi_{i a r}, & & a \in A, \quad i \in I: M_{a} \neq \emptyset, \\
y_{a} & =\underline{y}_{a}+\sum_{r \in M_{a}} r \zeta_{a r}, \quad \sum_{r \in M_{a}} \zeta_{a r}=1, & & a \in A: M_{a} \neq \emptyset, \\
\underline{q}_{i a} \zeta_{a r} & \leq \xi_{i a r} \leq \bar{q}_{i a} \zeta_{a r}, & & a \in A, \quad i \in I, \quad r \in M_{a}, \\
x_{i a} & =q_{i a} \underline{y}_{a}+\sum_{r \in N_{a}} 2^{r} \xi_{i a r}, & & a \in A, \quad i \in I: \quad N_{a} \neq \emptyset, \\
y_{a} & =\underline{y}_{a}+\sum_{r \in N_{a}} 2^{r} \zeta_{a r} \leq \bar{y}_{a}, & & a \in A: \quad N_{a} \neq \emptyset, \\
\xi_{i a r} & \geq \max \left\{\underline{q}_{i a} \zeta_{a r}, q_{i a}+\bar{q}_{i a}\left(\zeta_{a r}-1\right)\right\}, & & a \in A, \quad i \in I, \quad r \in N_{a}, \\
\xi_{i a r} & \leq \min \left\{\bar{q}_{i a} \zeta_{a r}, q_{i a}+\underline{q}_{i a}\left(\zeta_{a r}-1\right)\right\}, & & a \in A, \quad i \in I, \quad r \in N_{a},
\end{aligned}
$$

where $\underline{q}_{i a}, \bar{q}_{i a}, \underline{y}_{a}$ and $\bar{y}_{a}$ are the lower and upper bounds on $q_{i a}$ and $y_{a}$, respectively. For all $a \in A$, we set $M_{a}=\left\{0, \ldots, \bar{y}_{a}-\underline{y}_{a}\right\}$ resp. $N_{a}=\left\{0, \ldots,\left\lfloor\log _{2}\left(\bar{y}_{a}-\underline{y}_{a}\right)\right\rfloor\right\}$ if $\bar{y}_{a}-\underline{y}_{a} \geq 1$, and $M_{a}=\emptyset$ resp. $N_{a}=\emptyset$ if $\bar{y}_{a}=\underline{y}_{a}$. A trivial choice of the lower and upper bounds is $\left[\underline{q}_{i a}, \bar{q}_{i a}\right]=[0,1]$ and $\left[\underline{y}_{a}, \bar{y}_{a}\right]=\left[L_{a}^{A}, U_{a}^{A}\right]$. However, these may be tightened in a preprocessing step, the importance of which is evident in the unary and binary expansions [D-U] and [D-B]: the tightness of bounds determines the number of binary variables $\zeta_{a r}$ that need to be introduced. Note that $[C]$ and [D] are MINLPs, while [D-U] and [D-B] are MILPs.

\section{COMPUTATIONAL RESULTS AND CONCLUSION}

Our industry partner provided us with a data set representing supply and demand data (including quality specifications and contractual bonuses and penalties) of a real life mining company for the time horizon of two years. We split the data into problem instances of years, half-years and quarters. There are two run-ofmine stockpiles (i.e., $|S|=2$ ) with lower and upper bounds of $L_{1}^{S}=L_{2}^{S}=2 M, U_{1}^{S}=7 M, U_{2}^{S}=8 M$, and there are four qualities: ash, moisture, sulfur and volatile matter (i.e., $|K|=4$ ). Problem sizes for the 14 instances vary between $|T| \in\{10, \ldots, 22\}$ for quarterly, $|T| \in\{24, \ldots, 42\}$ for half-yearly and $|T| \in\{54,80\}$ for yearly instances. We used AMPL to model the different formulations and solved every instance for every formulation. The MINLPs [C] and [D] were solved with SCIP 3.0..$^{1}$ which we linked to CPLEX 12.6.0. $2^{2}$ as the LP solver and to Ipopt 3.10 $0^{3}$ as the NLP solver. We solved the MILPs [D-U] and [D-B] with CPLEX 12.6.0.0. Yearly instances had a time limit of 60 minutes, half-yearly instances had 30 minutes, and quarterly instances had 15 minutes. All computations were carried out on a Dell PowerEdge R710 with dual hex core 3.06GHz Intel Xeon X5675 processors and 96GB RAM, running Red Hat Enterprise Linux 6 and using a single thread. Let $z[f, i, M]$ denote the optimal objective function value of problem $[f], f \in\{\mathrm{C}, \mathrm{D}, \mathrm{D}-\mathrm{U}, \mathrm{D}-\mathrm{B}\}$, for instance $i$ and flow multiple $M$. It is clear that $z[\mathrm{C}, i, M] \leq z[\mathrm{D}, i, M]=$ $z[\mathrm{D}-\mathrm{U}, i, M]=z[\mathrm{D}-\mathrm{B}, i, M]$ for all $i$ and $M$. Comparing the best known lower bounds for the continuous and upper bounds for the discrete flow problems, we calculated the relative gap between the two as

$$
\operatorname{gap}(i, M)=\frac{\min \{\bar{z}[\mathrm{D}, i, M], \bar{z}[\mathrm{D}-\mathrm{U}, i, M], \bar{z}[\mathrm{D}-\mathrm{B}, i, M]\}-\underline{z}[\mathrm{C}, i, M]}{\underline{z}[\mathrm{C}, i, M]},
$$

where $\bar{z}[f, i, M]$ is the best upper and $\underline{z}[f, i, M]$ is the best lower bound on $z[f, i, M]$ that is found within the instance-specific time limit. If $\bar{z}[\mathrm{D}, i, M]=\bar{z}[\mathrm{D}-\mathrm{U}, i, M]=\bar{z}[\mathrm{D}-\mathrm{B}, i, M]=\infty$ (i.e., no upper bound was found for the discrete flow problem) or $\underline{z}[\mathrm{C}, i, M]=-\infty$ (i.e., no lower bound was found for the continuous flow problem), then $\operatorname{gap}(i, M)$ is not defined. Considering only the $(i, M)$-pairs for which gap $(i, M)$ is defined, the gaps for yearly instances are in [1.60\%,9.94\%], for half-yearly ones in $[0.13 \%, 4.57 \%]$ and for quarterly ones in $[0.04 \%, 4.25 \%]$. Computational results for $M \in\{8,4,2\}$ kt are shown in Table 2. We also solved instances for half and quarter trainloads to see if [D-B] performs better than [D-U] if the $y$ variables

\footnotetext{
${ }^{1}$ SCIP (scip.zib.de) is a non-commercial, global solver for MILP and MINLP, and our solver for [C] and [D].

${ }^{2}$ CPLEX (cplex.com) is a commercial, global solver for MILP and quadratic programming (QP), and our solver for [D-U] and [D-B].

${ }^{3}$ Ipopt (coin-or.org/lpopt) is an open source, local solver for NLP. The solver implements an interior point line search filter method to find local solutions. We use Ipopt in SCIP to find local solutions fast.
} 
Table 2. Computational results for $M \in\{8,4,2\} \mathrm{kt}$. If an instance could be solved within its time limit, we report the total solve time in seconds, otherwise we report the relative gap between the upper and lower bound in percent in brackets.

\begin{tabular}{|c|c|c|c|c|c|c|c|c|c|c|c|c|}
\hline \multirow[b]{2}{*}{ Instance } & \multicolumn{4}{|c|}{$M=8 \mathrm{kt}$} & \multicolumn{4}{|c|}{$M=4 \mathrm{kt}$} & \multicolumn{4}{|c|}{$M=2 \mathrm{kt}$} \\
\hline & {$[\mathrm{C}]$} & [D] & {$[\mathrm{D}-\mathrm{U}]$} & [D-B] & {$[\mathrm{C}]$} & [D] & {$[\mathrm{D}-\mathrm{U}]$} & [D-B] & {$[\mathrm{C}]$} & [D] & {$[\mathrm{D}-\mathrm{U}]$} & [D-B] \\
\hline Year 1 & $(\infty)$ & $(8.44)$ & (1.74) & $(3.82)$ & $(\infty)$ & $(9.61)$ & $(0.00)$ & (6.21) & (2.40) & $(4.85)$ & $(\infty)$ & $(\infty)$ \\
\hline Half-year 1 & (1.92) & $(0.60)$ & 1072.02 & 973.89 & $(3.35)$ & (4.33) & $(0.76)$ & (2.17) & (1.44) & $(2.28)$ & (1.27) & (1.44) \\
\hline Quarter 1 & $(0.15)$ & 7.70 & 3.91 & 4.64 & $(0.63)$ & 77.22 & 27.72 & 29.70 & $(0.47)$ & 365.04 & 189.79 & 376.52 \\
\hline Quarter 2 & $(0.12)$ & 22.81 & 14.44 & 12.60 & $(0.42)$ & 579.48 & 361.19 & 679.32 & $(0.34)$ & $(0.26)$ & $(0.06)$ & $(\mathbf{0 . 0 3})$ \\
\hline Half-year 2 & $(\infty)$ & 415.43 & 52.51 & 231.83 & (1.17) & (1.04) & $(0.97)$ & (1.48) & $(\infty)$ & (1.77) & (1.92) & (2.36) \\
\hline Quarter 3 & $(0.01)$ & 2.19 & 4.38 & 3.93 & $(0.05)$ & 10.49 & 17.10 & 10.25 & $(\infty)$ & 119.29 & 272.60 & 47.00 \\
\hline Quarter 4 & 688.62 & 6.04 & 1.82 & 3.88 & $(0.22)$ & 101.37 & 31.73 & 52.99 & $(\infty)$ & 357.00 & 152.21 & 350.21 \\
\hline Year 2 & $(\infty)$ & $(\infty)$ & 1752.94 & 3556.30 & (1.14) & $(\infty)$ & $(0.62)$ & (1.37) & $(\infty)$ & (1.81) & (0.74) & (1.54) \\
\hline Half-year 1 & $(0.66)$ & 72.54 & 16.30 & 29.13 & $(0.89)$ & 1243.82 & 174.17 & 727.81 & $(0.59)$ & $(0.91)$ & 1766.16 & $(0.32)$ \\
\hline Quarter 1 & $(0.04)$ & 2.87 & 2.85 & 3.54 & $(0.14)$ & 2.92 & 10.22 & 14.16 & $(0.24)$ & $(0.00)$ & 43.31 & 189.39 \\
\hline Quarter 2 & 61.61 & 0.33 & 0.49 & 0.71 & $(0.02)$ & 2.18 & 2.06 & 2.33 & 71.24 & 1.17 & 5.90 & 7.87 \\
\hline Half-year 2 & $(0.11)$ & 7.59 & 6.05 & 6.20 & $(0.21)$ & 69.25 & 27.18 & 98.54 & $(0.13)$ & 606.69 & 1752.79 & 679.21 \\
\hline Quarter 3 & 10.36 & 0.32 & 0.54 & 0.69 & 407.20 & 0.49 & 1.28 & 0.71 & 137.01 & 0.55 & 1.81 & 1.57 \\
\hline Quarter 4 & $(0.01)$ & 3.00 & 1.44 & 1.50 & $(0.04)$ & 6.07 & 5.16 & 8.14 & $(\infty)$ & 15.28 & 24.53 & 89.99 \\
\hline
\end{tabular}

can take more discrete values. We see that the discretized problems [D], [D-U] and [D-B] perform much better than the continuous problem [C]. In 27 out of 42 runs, [D-U] outperforms [D] and [D-B]. There are only 9 runs in which [D] performs best, and only 5 runs in which [D-B] performs best - despite the fact that the formulation has far fewer binary variables than [D-U]. [C] only wins once, and that is due to [D-U] and [D-B] not finding any upper bounds. We can conclude that it is highly advantageous to discretize flow variables (if the application allows it), and that the exact MILP linearizations perform significantly better than the MINLPs.

\section{ACKNOWLEDGEMENT}

This research was supported by the ARC Linkage Grant no. LP110200524, Hunter Valley Coal Chain Coordinator (hvccc.com.au) and Triple Point Technology (tpt.com). We thank the two anonymous referees for their comments which helped to improve the quality of the paper.

\section{REFERENCES}

Alfaki, M. and D. Haugland (2013). A multi-commodity flow formulation for the generalized pooling problem. Journal of Global Optimization 56(3), 917-937.

Boland, N., T. Kalinowski, and F. Rigterink (2015a). New multi-commodity flow formulations for the pooling problem. Optimization Online. e-print ID 4959.

Boland, N., T. Kalinowski, F. Rigterink, and M. Savelsbergh (2015b). A special case of the generalized pooling problem arising in the mining industry. Optimization Online. e-print ID 5025.

Boland, N., M. Savelsbergh, and H. Waterer (2015c). A decision support tool for generating shipping data for the Hunter Valley coal chain. Computers \& Operations Research 53, 54-67.

Gupte, A., S. Ahmed, M. S. Cheon, and S. S. Dey (2013). Solving Mixed Integer Bilinear Problems Using MILP Formulations. SIAM Journal on Optimization 23(2), 721-744.

Kozan, E. and S. Q. Liu (2012). A demand-responsive decision support system for coal transportation. Decision Support Systems 54(1), 665-680.

Liu, S. Q. and E. Kozan (2011). Optimising a coal rail network under capacity constraints. Flexible Services and Manufacturing Journal 23(2), 90-110.

Robinson, G. K. (2004). How much would a blending stockpile reduce variation? Chemometrics and Intelligent Laboratory Systems 74(1), 121-133.

Singh, G., R. García-Flores, A. Ernst, P. Welgama, M. Zhang, and K. Munday (2014). Medium-Term Rail Scheduling for an Iron Ore Mining Company. Interfaces 44(2), 222-240.

Singh, G., D. Sier, A. T. Ernst, O. Gavriliouk, R. Oyston, T. Giles, and P. Welgama (2012). A mixed integer programming model for long term capacity expansion planning: A case study from The Hunter Valley Coal Chain. European Journal of Operational Research 220(1), 210-224.

Thomas, A., G. Singh, M. Krishnamoorthy, and J. Venkateswaran (2013). Distributed optimisation method for multi-resource constrained scheduling in coal supply chains. International Journal of Production Research 51(9), 2740-2759. 